die, die einen Vorteil zeigte: 115 vs. 51 Monate medianes Überleben zugunsten der Chelierung. Nun gibt es zusätzlich eine qualitativ höherwertige Matched-PairAnalyse mit 186 MDS-Patienten der Universität Düsseldorf mit ähnlichem Ergebnis: 75 vs. 49 Monate medianes Überleben zugunsten der Eisenentleerung, die früher mit dem täglich s.c. zu infundierenden Deferoxamin (Desferal ${ }^{\circledR}$ ) erfolgte und heute mit dem oral einzunehmenden Deferasirox (Exjade ${ }^{\circledR}$ ) durchgeführt wird.

Folgende Patienten profitieren laut Gering von der Chelierung: Patienten mit relativ günstigen MDS-Stadien und mehrjähriger Lebenserwartung, mit dokumentiertem stabilem Verlauf, mit Ferritinwerten über $1000 \mathrm{ng} / \mathrm{ml}$ oder anderem Nachweis von Organeisenüberladung sowie Kandidaten für die allogene Transplantation.

- Dr. med. Dirk Einecke

Quelle: Press Round Table Discussion,

29. Deutscher Krebskongress, Berlin,

Februar 2010 (Veranstalter: Novartis Oncology)

Aldosteronblockade bei Herzinsuffizienz nach Myokardinfarkt

\title{
Früh starten, langfristig beibehalten
}

— Klinische Zeichen der Herzschwäche in den ersten Tagen nach Myokardinfarkt signalisieren eine schlechte Prognose - selbst dann, wenn die Herzschwäche nur diskret und vorübergehend ist. Als Grund hierfür nannte Prof. Johann Bauersachs, Würzburg, den durch die nachlassende Schlagkraft induzierten Aldosteronanstieg. Verbessern lässt sich die Prognose mit einer frühen Aldosteronblockade. Das belegt die EPHESUS-Studie, in der 6632 Postinfarktpatienten mit Symptomen der Herzinsuffizienz innerhalb von 3 bis 14 Tagen additiv zur Standardtherapie Eplerenon oder Placebo erhielten.

Bereits nach 30 Tagen war die Überlebensrate mit Eplerenon um 31\% höher als mit Placebo. Die kardiovaskuläre Sterblichkeit lag um 32\% geringer und der plötzliche Herztod sogar um 37\%. Dabei hatten die Patienten, bei denen die Therapie zwischen Tag drei und sieben begann, eine noch bessere Prognose als die Patienten mit späterem Behandlungsbeginn. Der günstige Effekt war anhaltend: Nach 16 Monaten - bis hin zu 33 Monaten - lag die Gesamtmortalität mit Eplerenon um 15\% niedriger als mit Placebo. Das Herztodrisiko war um $21 \%$ reduziert, in der Subgruppe der Infarktpatienten mit einer Ejektionsfraktion unter $30 \%$ sogar um $33 \%$.

Diese Daten sprechen dafür, bei Patienten mit Zeichen der Herzinsuffizienz in den ersten Tagen nach Myokardinfarkt so früh wie möglich Eplerenon einzusetzen (zugelassen ab Tag drei) und dauerhaft als festen Baustein in die Sekundärprävention einzubauen.

- Dr. med. Kirsten Westphal

Quelle: Satellitensymposium, DGK-Jahres-

tagung, Mannheim, 9. April 2010

(Veranstalter: Pfizer)

\section{Osteoporosetherapie}

\section{Inhomogenität schafft Stabilität}

- Um bei Osteoporose die größtmögliche Stabilität des Knochens zurückzugewinnen, ist eine Restrukturierung anzustreben, die dem physiologischen Knochenumsatz nahekommt. Dies ist Experten zufolge mit Strontiumranelat weitgehend möglich.

Alle Verformungen des Knochens - sie finden im Alltag ständig statt - führen zu kleinen Rissen, sogenannten Microdamages, erklärte Prof. Dr. F. Jacob, Würzburg. Eine inhomogene Struktur des Knochens sorgt dafür, dass sich die Risse nicht weiter ausdehnen können. Voraussetzungen dafür sind eine ausgewogene Kalziumeinlagerung sowie ein möglichst physiologisches Gleichgewicht zwischen Knochenabbau und -aufbau. „Nur bei einer Interaktion von Osteoklasten und Osteoblasten können Mikrofrakturen narbenlos verheilen.“

\section{Jahrelanger Frakturschutz gut belegt} Strontiumranelat (Protelos ${ }^{\circledR}$ ) wirkt zugleich hemmend auf die Osteoklasten und stimulierend auf die Osteoblasten und sorgt auf diese Weise für einen ausbalancierten Knochenstoffwechsel. Die Mineralisation des Knochens bleibt erhalten, da weder übermäßige antiresorptive Effekte noch allzu starke osteoanabole Impulse auftreten, wie Prof. Dr. Andreas Kurth, Mainz, darlegte. Kurth wies auch darauf hin, dass Strontiumranelat bei Hüftgelenkersatz die Mikroarchitektur des Knochens rund um Implantate entscheidend verbessern kann. Dank der erhöhten Ausreißfestigkeit kommt es seltener zu einer Lockerung der Endoprothese. Daten aus Tierstudien ließen diese Schlussfolgerungen zu.

Die fraktursenkende Wirkung von Strontiumranelat ist laut Kurth besonders gut belegt. In prospektiven Studien über fünf Jahre wurde eine signifikante Reduktion sowohl von Wirbelkörperfrakturen ($24 \%)$ als auch von Hüftfrakturen (-43\%) nachgewiesen. Die anhaltende Wirksamkeit des Osteoporosetherapeutikums bestätigte sich auch in einer offenen Verlängerungsstudie über acht Jahre. Voraussetzung für eine langfristige Therapie ist eine gute Compliance. Dass diese bei Strontiumranelat-Behandlung gegeben ist, lässt sich einer europäischen Kohortenstudie entnehmen: Von über 13000 Patienten waren nach einem Jahr noch $80 \%$ im Therapiearm im Vergleich zu nur 50\% bei einer Bisphosphonat-Behandlung.

- Reimund Freye

Quelle: Satellitensymposium „Knackpunkt Osteoporose“, Jahrestagung der Süddt. Orthopäden, Baden-Baden, 30. April 2010 (Veranstalter: Servier) 